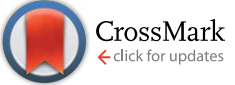

Cite this: RSC Adv., 2015, 5, 93666

Received 7th September 2015 Accepted 23rd October 2015

DOI: $10.1039 / c 5 r a 18296 d$

www.rsc.org/advances

\section{Modulating the elution of antibiotics from nanospongy titanium surfaces with a $\mathrm{pH}$-sensitive coating}

\author{
Selya Amrani, ${ }^{a}$ Aman Atwal $^{\text {ab }}$ and Fabio Variola*ac
}

Metals currently used for prosthetic reconstructions enjoy a relatively good success rate, but post-surgical infections still remain an important challenge. In addition, there are still no such metals that are able to respond to any deterioration of their relationship with the host tissue, in particular to an acidification of the local environment, an event associated to bone remodeling, tissue inflammation and bacterial infection. Distinctive from previous work that employed anodization of titanium to engender nanotubular structures, we exploited this technique to create a nanospongy surface that behaved as a non-eroding drug-eluting template for the extended release of vancomycin, a model antibiotic. Successively, as a proof of concept, we employed a chitosan-poly(ethylene glycol) (PEG) coating to provide $\mathrm{pH}$-dependent release kinetics of vancomycin molecules stored in the underlying 3-dimensional network of nanometric pores. A physicochemical characterization of the polymeric blend by ATR-FTIR and DPFM-AFM unveiled its morphological and nanomechanical characteristics and permitted to link them to its stability and swelling behavior in aqueous solutions at three different pHs. This study demonstrates the ability of nanospongy titanium surfaces to provide extended elution of vancomycin, one of the most effective antibiotics against Gram-positive bacteria, for over one week, thus becoming a valid alternative to existing drug-eluting metallic platforms. In addition, our results show how the elution profiles can be modulated to respond to an acidification of the surrounding environment by exploiting uncross-linked and cross-linked chitosan-PEG coatings, ultimately paving the way for their broader use as a versatile coating for nanoporous drug-delivery platforms.

\section{Introduction}

Biocompatible metals currently used for prosthetic reconstructions enjoy a relatively good success rate, but they still need significant improvement with respect to their capacity to secure rapid and long-lasting integration in tissues, especially in aging patients and in individuals with compromised health status. ${ }^{1-3}$ In addition, the risk of infection remains an important complication for $2-5 \%$ of surgeries, with effects that range from superficial skin conditions to life-threatening sepsis. ${ }^{4,5}$ The key to respond to the need of better performing biomedical implants is the use of functional materials capable of controlling the biological events that ultimately dictate the outcome of implanted devices. ${ }^{6}$ In this context, it has been shown that nanoporous and nanotubular titanium surfaces engendered by

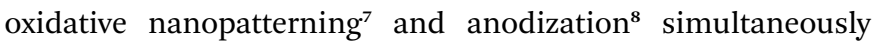
provide beneficial effects to adhering cells and inherently discourage bacterial adhesion and growth. ${ }^{9-13}$ Nanoporous/

${ }^{a}$ Department of Mechanical Engineering, University of Ottawa, Canada. E-mail: fabio. variola@uottawa.ca

${ }^{b}$ Department of Biopharmaceutical Sciences, University of Ottawa, Canada

${ }^{c}$ Department of Physics, University of Ottawa, Canada nanotubular surfaces have also proven to be efficient eluting platforms, able of storing bioactive agents (e.g. growth factors, antibiotics) and modulating their release over time, thereby achieving synergistic abilities that conjugate direct physicochemical cueing and drug-delivery. ${ }^{14,15}$ Although effective, these nanoengineered surfaces are not yet able to sense any deterioration of their relationship with the surrounding biological environment, in particular whether the release of the stored bioactive agents is needed to respond to specific cues from the body. In order to endow drug-delivery platforms with the capacity of discerning whether a certain reaction is required in response to a particular event (e.g. bone resorption, development of a bacterial infection), researchers have aimed at designing polymeric coatings capable of providing a 'gated' release of drugs triggered by environmental stimuli. ${ }^{16}$

While aiming at a more sophisticated ability to sense and respond to environmental changes (e.g. variations in $\mathrm{pH}$, temperature, light, enzymatic activity), researchers have combined different polymers to achieve biocompatible blends increasingly more receptive to physicochemical cues. ${ }^{17-19}$ In this context, biopolymers that respond to $\mathrm{pH}$ variations from physiological (7.2-7.4) to acid values are very attractive for drugdelivery applications. In fact, an acidification of the local 
environment has been associated with bone remodeling, ${ }^{20,21}$ tissue inflammation ${ }^{22}$ and bacterial activity. ${ }^{23-25}$ Therefore, pH-sensitive biopolymers are expected to make a perfect candidate as environmentally sensitive switch to initiate and control the release of pharmaceutical agents (e.g. bone resorption inhibitors to combat bone loss, anti-inflammatory agents to control the inflammatory response and/or antibiotics to fight the onset of bacterial infection) only when instructed by the body. In the case of infection, biopolymers that dissolve and degrade with $\mathrm{pH}$-dependent rates supply additional antifouling capacities by contrasting the initial bacterial attachment. ${ }^{16}$

In this study, we aimed at merging the morphological characteristics of nanoporous and nanotubular titanium, two effective drug-eluting substrates, ${ }^{7,8}$ to engender a "hybrid" nanospongy surface that promises to integrate their benefits (i.e. ease of fabrication, open porosity that facilitates loading, significant loading capacity, satisfactory fatigue resistance and short-term mechanical stability) ${ }^{\mathbf{7}, \mathbf{8 , 1 0 , 1 4 , 1 5 , 2 6}}$ while limiting their disadvantages (i.e. relatively low loading capacity and potential delamination/degradation in the biological environment). ${ }^{\mathbf{1 4 , 1 5}}$ We then assessed, for the first time, the potential of this surface as a non-eroding drug-delivery template by quantifying the elution kinetics of vancomycin, one of the most effective antibiotics against Gram-positive bacteria. ${ }^{27}$

In order to achieve the ability to respond to an acidification of the surrounding environment and provide $\mathrm{pH}$-dependent elution profiles, as a proof of concept we successively coated vancomycinloaded nanospongy surfaces with a chitosan-polyethylene glycol (PEG) film. It should be noted that although chitosan-PEG blends have been previously used as drug-delivery systems for bioactive agents incorporated in the polymeric network, ${ }^{28}$ to date their potential as a $\mathrm{pH}$-sensitive filter capable of regulating the elution from underlying non-eroding surfaces still needed to be validated. Previous work with nanoporous titanium showed in fact either a $\mathrm{pH}$-dependent release achieved by incorporating the drugs into a dissolving coating (hence, no filtering effects by the polymeric film) ${ }^{16}$ or filtering effects with no environmental responsiveness. ${ }^{29}$ By capitalizing on these studies, we contributed to the development of an effective metal-biopolymer system by demonstrating the pH-dependent elution capacities of chitosanPEG coated nanospongy surfaces. To this end, we assessed the release kinetics at three different $\mathrm{pHs}(7.4,5$ and 3$)$ to incorporate all those events (e.g. bacterial infection, bone and tissue remodeling, inflammation) which are characterized by a localized acidification of the physiological microenvironment. In order to improve the stability of the blend and increase its performances, we also employed genipin, a naturally occurring cross-linker for chitosan hydrogels. ${ }^{30,31}$ Attenuated Total Reflection FourierTransform Infrared Spectroscopy (ATR-FTIR) and Digital Pulsed Force Mode Atomic Force Microscopy (DPFM-AFM) were used to investigate the physicochemical properties of the coating.

This study demonstrates that the drug eluting capacities of nanospongy titanium surfaces can be engineered to respond to an acidification of the environment by exploiting a $\mathrm{pH}$-sensitive coating capable of regulating the release of antibiotics stored in the underlying nanoconfined volumes. Our findings highlight the potential of a chitosan-PEG blend to act as a "molecular filter" that provides one extra level of synergistic control of the elution profiles from non-eroding nanoporous templates, paving the way for its broader use in those applications requiring drug-eluting metallic substrates able to respond to localized environmental changes.

\section{Materials and methods}

\section{Anodization}

Commercially pure titanium (CP-Ti) of ASTM Grade 2 titanium disks (Firmetal Co., Ltd., Shanghai, China), $12 \mathrm{~mm}$ in diameter and $1 \mathrm{~mm}$ in thickness, were mechanically polished with a 2-step process by the supplier. As-received disks were first cleaned in toluene in an ultrasonic bath for 15 min then rinsed in deionized water. A solution of $0.5 \% \mathrm{HF}$ (Fisher Scientific, USA) was used as the electrolyte in a 2-electrode cell, where the anode was the titanium disk and the cathode a $25 \times 25 \mathrm{~mm}$ platinum foil (Alfa Aesar, USA). Samples were anodized under constant voltage ( $35 \mathrm{~V}$, corresponding to a current of $10 \mathrm{~mA}$ ) for 15 minutes to engender nanospongy surfaces.

\section{Drug loading}

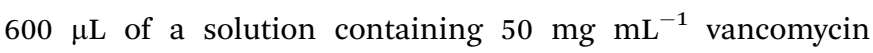
hydrochloride (Sigma Aldrich, USA) in 10\% ethanol were pipetted onto nanospongy titanium disks placed inside custommade cylindrical holders. Ethanol was used to facilitate the penetration of the solution into the 3-dimensional network of nanopores by reducing the surface tension of the water. ${ }^{\mathbf{1 4}}$ Vancomycin-loaded disks were dried in a vacuum-oven at $45{ }^{\circ} \mathrm{C}$ for 12 hours. After removing the excess of vancomycin with a blade, the samples were gently rinsed with deionized water to remove vancomycin residues, gently blotted and dried overnight in a vacuum-oven. Samples were finally weighted with a high precision scale ( $0.01 \mathrm{mg}$ sensitivity) to determine the mass of vancomycin stored in the nanoconfined pores of nanospongy surfaces.

\section{Proton nuclear magnetic resonance (H-NMR)}

In order to assess whether the vacuum drying protocol had any effect on the antibiotic's structure, we carried out proton nuclear magnetic resonance analysis. Two samples were considered: (i) the as-received vancomycin hydrochloride powder and (ii) the antibiotic powder recovered from a $50 \mathrm{mg} \mathrm{mL}^{-1}$ solution of vancomycin in $10 \%$ ethanol dried in vacuum at $45{ }^{\circ} \mathrm{C}$ for 12 hours. Approximately $5 \mathrm{mg}$ of each sample were dissolved in DMSO-d6 in a glass vial. The solutions were transferred to standard $5 \mathrm{~mm}$ NMR tubes and analyzed with an Avance III HD $600 \mathrm{MHz}$ NMR spectrometer equipped with a cryoprobe (Bruker, USA). Data were collected using a standard one-pulse sequence. A 30-degree pulse, 4 second acquisition time and a $10 \mathrm{msec}$ recycle delay were used. Sixteen transients were signal-averaged and Fourier-transformed without apodization to produce the NMR spectra.

\section{Chitosan-polyethylene glycol coatings}

A mixture of medium molecular weight chitosan - hereafter indicated as $\mathrm{CH}$ - and a low molecular weight (Mn 400) 
polyethylene glycol - hereafter indicated as PEG - (both purchased from Sigma Aldrich, USA) with a 10: 90 weight ratio was diluted to a concentration of $100 \mathrm{mg} \mathrm{mL}^{-1}$ in $1 \%$ acetic acid. The choice of such composition was motivated by the following factors: (i) chitosan-PEG blends show superior properties (e.g. stability in water, mechanical properties) than the single components alone, ${ }^{32,33}$ (ii) their stability, permeability and swelling characteristics are $\mathrm{pH}$-sensitive, ${ }^{28,33}$ (iii) the degree of pH-dependent swelling increases with the PEG content, ${ }^{33}$ (iv) a preparatory study with three different compositions, i.e. $10: 90$, $50: 50$ and $90: 10$ confirmed that the $10: 90$ ratio provided the most marked pH-dependent swelling behaviour (data not shown), (v) the diffusion rate of molecules through chitosan-PEG membranes is affected by the $\mathrm{pH}$ and the degree of cross-linking, $^{28}$ (vi) the low chitosan-to-PEG ratio provides additional antifouling properties by contrasting initial bacterial adhesion via dissolution of PEG in water ${ }^{32}$ and (vii) low-molecular weight PEG has demonstrated to possesses anti-inflammatory properties and to improve survival during sepsis, ${ }^{34}$ a characteristic that further strengthens the antibacterial properties of the drugeluting platform developed in this study. Taken together, these characteristics made the 10 : 90 chitosan-PEG blend the optimal candidate to endow nanospongy surfaces with $\mathrm{pH}$-dependent drug-eluting abilities for antibacterial applications.

Successively, 0.5 wt\% genipin (Sigma Aldrich, USA), here after indicated as GEN, was added to the CH:PEG solution under continuous stirring at room temperature for $2 \mathrm{~h}$. $250 \mu \mathrm{L}$ of the resulting solution were pipetted onto the vancomycin-loaded disks in order to obtain 15-20 $\mathrm{mg}$ of the blend, measured after immersion in $100 \mathrm{~mL}$ of methanol for $24 \mathrm{~h}$ to remove the acetic $\operatorname{acid}^{33}$ and vacuum-drying at $45{ }^{\circ} \mathrm{C}$ for 12 hours. The amount of the solution deposited on the disks was optimized to provide a homogeneous coverage of the surface.

\section{Swelling and stability of the coatings}

In order to determine the stability and swelling characteristics, CH:PEG coated disks were accurately weighed before $\left(W_{\mathrm{dd}}\right)$ and after $\left(W_{\text {id }}\right)$ the deposition of the polymeric film by using a high precision scale with a $0.01 \mathrm{mg}$ sensitivity. Aqueous solutions at three different $\mathrm{pH}(3,5$ and 7.4) were prepared, in which the coated disks were immersed during varying time intervals (i.e. $0.25,0.5,0.75,1,2,3,6,12,18,24,72$ and $168 \mathrm{~h}$ ). Five disks/time point were used for swelling and stability experiments for each of the 3 pHs. Each disk was weighted three times to ensure consistency of the readings.

To determine the degree of swelling, the disks were removed from the solutions and weighted after careful blotting with a filter paper to remove the excess of surface water $\left(W_{\mathrm{fw}}\right)$. They were successively dried for $24 \mathrm{~h}$ under vacuum at $45{ }^{\circ} \mathrm{C}$ and weighted again $\left(W_{\mathrm{fd}}\right)$. The weight uptake percentage of the coatings was expressed by the following equation: $E_{\mathrm{sw}}=\left[\left(W_{\mathrm{fw}}-W_{\mathrm{fd}}\right) /\left(W_{\mathrm{fd}}-\right.\right.$ $\left.\left.W_{\mathrm{dd}}\right)\right] \times 100$, where $E_{\mathrm{sw}}$ is the percentage of water of adsorption of the film at equilibrium. The stability of the coating at the three different pHs was then calculated according to the following equation: $S=\left[\left(W_{\mathrm{fd}}-W_{\mathrm{dd}} / W_{\mathrm{id}}-W_{\mathrm{dd}}\right)\right] \times 100$, where $S$ is the percentage of the coating remaining after immersion.

\section{Scanning electron (SEM) and digital pulsed force atomic force (DPFM-AFM) microscopy}

A JSM-7500F Field Emission Scanning Electron Microscope (FESEM, JEOL, Japan) was used to image nanospongy titanium samples. Before each elution experiments, anodized disks were imaged to validate the presence of the nanospongy surface. In addition, SEM imaging was used to ensure that our experimental protocol yielded a uniform coverage of the disk by the polymeric coating.

A WITec Alpha 300 integrated microscope (WITec, Germany) was used to collect AFM images of $\mathrm{CH}$ :PEG coated nanospongy disks in Digital Pulsed Force (DPFM) mode. This technique permitted to visualize the nanoscale surface morphology of the coating while simultaneously probing its nanomechanical properties. Three images were obtained from three different CH:PEG and CH:PEG + GEN samples by using a cantilever with a tetrahedral tip (tip radius less than $10 \mathrm{~nm}$ ) and a nominal spring constant of $0.2 \mathrm{~N} \mathrm{~m}^{-1}$ (Arrow ${ }^{\mathrm{TM}}$ FM Nanoworld, Switzerland). Each DPFM-AFM image contains $2.5 \times 10^{5}$ forcedistance curves. From these, we selected 20 curves per image (totalling 60 curves per condition), which were successively converted into force $v s$. indentation depth in order to calculate the stiffness values, according to a previously published procedure..$^{35,36}$

\section{Ultraviolet-visible (UV-VIS) spectroscopy}

Vancomycin-loaded disks were placed inside custom-made cylindrical holders. $300 \mu \mathrm{L}$ of aqueous solution at the 3 different pHs $(3,5,7.4)$ were added. The holder was sealed with parafilm to prevent evaporation and placed on a horizontal shaker at room temperature. Constant volume aliquots were collected at 10 time points. The absorbance of the vancomycin was measured at $280 \mathrm{~nm}$ by exploiting a UV-VIS spectrophotometer (Biotek Epoch, Fisher Scientific) ${ }^{\mathbf{1 4}}$ after having ensured that neither chitosan or PEG absorbs at this wavelength. All elution experiments were carried out in quintuplets. Equal amounts of solution were added every time the samples were taken to maintain a constant volume. The mass eluted at every time point was calculated based on the calibration curves obtained with known concentrations of the vancomycin in deionized water, and adjusted to account for variations in concentration due to the systematic removal and replacement of aliquots. In particular, the eluted mass in $20 \mu \mathrm{L}$ aliquots (measured by UV-VIS) taken at the time interval $t_{\mathrm{i}}$ was calculated by accounting for the mass removed from the $300 \mu \mathrm{L}$ volume system by the aliquot taken at the interval $t_{\mathrm{i}-1}$ (which was replaced with an equal amount of aqueous solution). ${ }^{\mathbf{1 4}}$

\section{Data fitting}

In the case of uncoated nanospongy surfaces, we applied the Korsmeyer-Peppas model $\left(M(t) / M_{\infty}=k t^{n}\right)$ to fit the portion of the elution curves for which the fraction of vancomycin eluted at time $t$ (i.e. $M(t) / M_{168 \mathrm{~h}}$ ) was below 0.6.37,38 In our calculations, $k$ (release rate constant) was the fitting parameter while $n$ (release exponent) was set to 0.5 , a value which is associated to a Fickian 
diffusion from non-eroding nanoporous surfaces. ${ }^{\mathbf{1 4 3 9 , 4 0}}$ To further characterize the elution kinetics of vancomycin until completion, we applied a model that accounts for a second phase characterized by the release of molecules through a series of interconnected channels, ${ }^{\mathbf{4 1}}$ resulting in the following comprehensive relationship: $M(t) / M_{\infty}=a+k t^{0.5}+b[1-\exp (-D t)]$.

\section{Attenuated total reflectance Fourier transform infrared (ATR- FTIR) spectroscopy}

Infrared analysis was carried out with a Bruker Tensor 37 FTIR spectrometer equipped with (RT) DLaTGS detector (Bruker, USA) and a platinum ATR accessory with a $2 \mathrm{~mm}$ diameter single reflection diamond crystal. Three $\mathrm{CH}$ :PEG coated disks samples per condition (i.e. with and without GEN) were analyzed. Spectroscopic information was collected in the 1000$1800 \mathrm{~cm}^{-1}$ range, with a $4 \mathrm{~cm}^{-1}$ resolution and 256 acquisitions per spectrum, using a bare titanium sample for reference. Infrared data were processed by using the OriginPro software and interpretation was carried out based on previous literature..$^{36,42,43}$ Spectra were normalized against the band centered at about $1095 \mathrm{~cm}^{-1}$.

\section{Results and discussion}

Fig. 1A displays the morphology of titanium surfaces after anodization in $0.5 \% \mathrm{HF}$ at $35 \mathrm{~V}$ for 15 minutes. Under these specific conditions, a transition from the well-known nanotubular morphology to a nanospongy structure occurred, giving rise to a 3-dimensional network of interconnected pores randomly distributed across the surface. Because of the complex and irregular nature of nanospongy surfaces (Fig. 1A), we employed image analysis (ImageJ ${ }^{\mathbf{4} 4}$ to quantify the dimension of only those nanopores which exhibited a well-defined circular morphology (i.e. circularity factor $>0.5$ ), revealing that their diameter is in the 50-100 nm range. Noteworthy, the conversion from nanotubes to the sponge-like structure is usually associated to more drastic anodization parameters, and was previously reported to be a result of the rotation rate of a titanium anode in a glycerol/water $/ \mathrm{NH}_{4} \mathrm{~F}$ electrolyte. ${ }^{45}$ Here, we show that nanospongy surfaces can be equally obtained in static conditions by using a $0.5 \%$ HF. In this context, diluted hydrofluoric acid is routinely used to generate arrays of nanotubes with controlled morphologies on titanium. ${ }^{\mathbf{8} 46}$ Like in the case of the glycerol/water/ $\mathrm{NH}_{4} \mathrm{~F}$ electrolyte, we remarked that
A

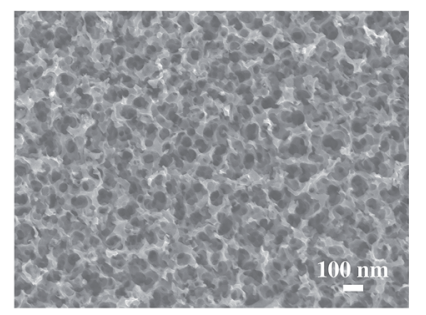

B

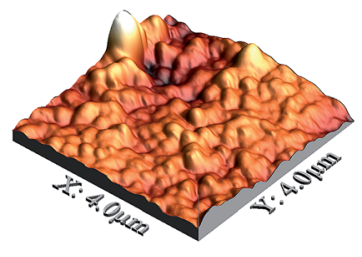

Fig. 1 (A) SEM and (B) AFM images of nanospongy titanium surfaces. nanospongy surfaces result from a more aggressive treatment with voltages greater than $30 \mathrm{~V}$. Conversely, for lower values (i.e. in the 5-20 $\mathrm{V}$ range), the characteristic voltage-dependent nanotubular morphologies are formed. ${ }^{46}$

SEM imaging was complemented by AFM investigation, which allowed us to close in on the features of the resulting surfaces. Fig. 1B displays a representative $4 \times 4 \mu^{2}$ AFM micrograph of treated samples, revealing the distinctive nanorough topography exhibiting a root-mean-square (RMS) roughness of $139 \pm 21 \mathrm{~nm}$. This value is significantly greater than those previously measured on nanoporous (i.e. $17-20 \mathrm{~nm})^{\mathbf{1 4 , 4 7}}$ and nanotubular (i.e. $25-77 \mathrm{~nm})^{8}$ surfaces, thereby indicating a more prominent 3-dimensional character.

In order to ensure that the drug-loading protocol did not alter the properties of vancomycin (a factor which could affect the UV-VIS readings as well as the antibiotic's efficiency for drug-delivery applications), we carried out H-NMR spectroscopic analysis. Fig. 2A shows that the spectra of vancomycin hydrochloride powder as received (blue line) and recovered after drying in vacuum at $45{ }^{\circ} \mathrm{C}$ for 12 hours (red line) are indistinguishable from one another, thereby demonstrating that the loading protocol did not impact the pristine properties of the antibiotic.

Successively, to determine the drug-release potential of the nanospongy surface, we monitored the release of vancomycin at various intervals ranging from 15 minutes to one week (168 hours), in order to operate within vancomycin in vitro's half-life (i.e. approximately 9 days) ${ }^{48}$ and link our study to potential in vivo antibacterial applications. ${ }^{14}$ We investigated the release kinetics at three different $\mathrm{pH}(7.4,5$ and 3$)$ in order to rule out any effect of the $\mathrm{pH}$ on the elution of vancomycin, thereby ensuring that the results obtained by applying the $\mathrm{CH}$ :PEG coating (Fig. $5 \mathrm{E}$ and F) are solely due to the pH-responsiveness of the blend. Fig. 2B displays the fraction of vancomycin eluted as a function of time, revealing two major aspects: (i) nanospongy surfaces release most of vancomycin (>95\%) after one week with a twophase profile and (ii) the elution kinetics are not affected by the $\mathrm{pH}$, since the three set of data points overlap significantly. These results, obtained by UV-VIS spectroscopy, were supported by direct weighting of vancomycin-loaded samples before and after elution. In fact, while the average mass loaded into nanospongy surfaces was $135 \pm 50 \mu \mathrm{g}$ (Fig. 2B, orange bar and shadowing, $W_{\mathrm{tL}}$ ), the total mass eluted (calculated by subtracting the mass left on each disk weighted at the end of the elution from the corresponding initial value) was $130 \pm 50 \mu \mathrm{g}$ (Fig. 2B, orange bar and shadowing, $W_{\mathrm{tE}}$ ), thereby confirming that more than $95 \%$ of vancomycin was released after one week. Noteworthy, the mass eluted determined by direct weighting is in very good agreement with the UV-VIS measurements, which quantified the average eluted mass to be $136 \pm 39 \mu \mathrm{g}$ after one week (Fig. 2B, green bar and shadowing, UV-VIS $\mathrm{E}_{\mathrm{E}}$. Fig. 2B displays the same curve in a logarithm scale in order to facilitate the comparison with the elution curves resulting from the use of the polymeric coating (Fig. 5E and F). When compared to nanoporous titanium surfaces generated by oxidative nanopatterning, nanospongy samples did not show a significant increase in the loading ability of vancomycin $(135 \pm 50$ vs. $106 \pm 60 \mu \mathrm{g}) .{ }^{14}$ However, the elution kinetics 
A

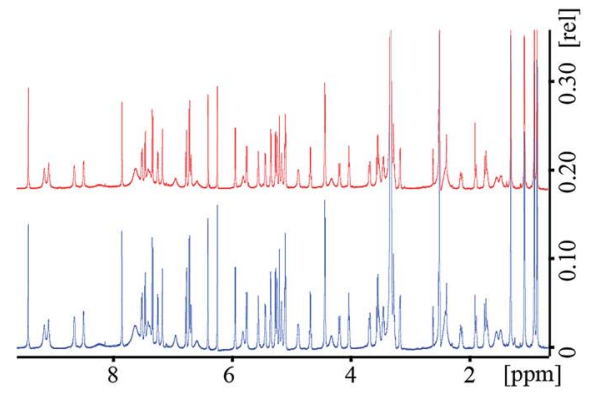

B

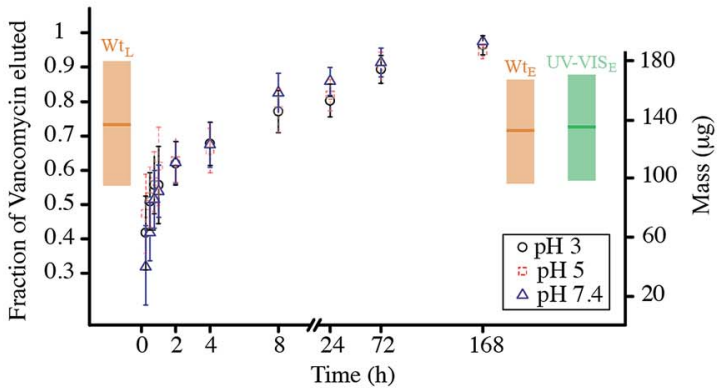

C

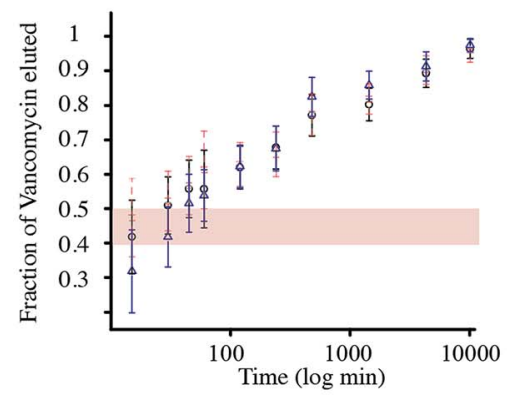

D

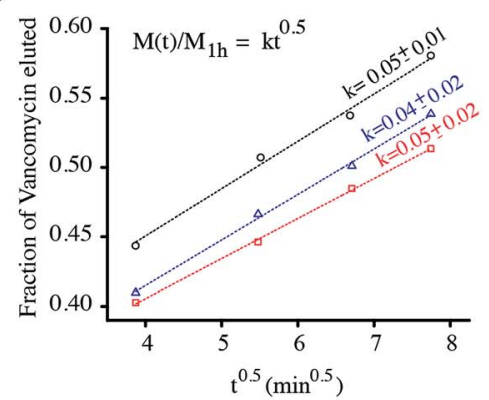

E

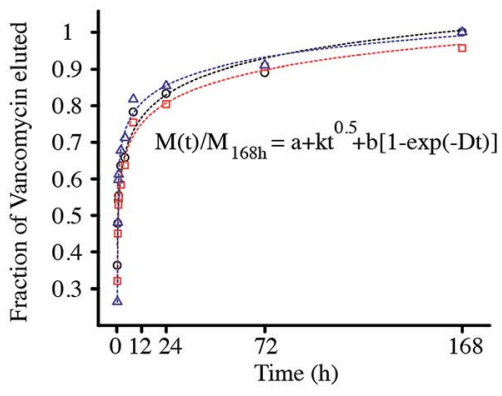

Fig. 2 (A) H-NMR spectra of vancomycin hydrochloride powder as received (blue line) and recovered after drying in vacuum at $45^{\circ} \mathrm{C}$ for 12 hours (red line). (B) Point-by-point curve of the fraction eluted of vancomycin from uncoated nanospongy surfaces ( $n=5)$. Data points are visualized as mean \pm standard deviation. To enhance clarity, the elution curve at $\mathrm{pH}=5$ (red dotted line) is shown with a transparency of $50 \%$. The orange and green bars and shadowing represent respectively the average value and standard deviation of the loaded (L) and eluted (E) mass quantified by weighting with a high sensitivity scale $\left(W_{t}\right)$ and by UV-VIS spectroscopy. (C) Representation of the point-by-point curve in a logarithmic scale. The red shadowing indicates the 40-50\% range of elution and was used as a visual reference for comparison. (D) Fitting of the initial portion of the elution curves with a Fickian model. (E) Elution of vancomycin fitted with a two-phase model.

resulted significantly slower and extended beyond one week, a finding that can be explained in light of the deeper and more complex 3-dimensional network of pores that characterized the anodic sponge-like structure. Conversely, when compared to conventional nanotubular templates (which proved to provide sustained elution of drugs beyond one month), ${ }^{39,40}$ nanospongy surfaces demonstrate their limitation, which most likely results from a reduced loading capacity. In this context, it should be noted that for antibacterial applications, a sustained elution of antibiotics to contrast the onset and development of bacterial infection may only be needed in the shorter term. In fact, postsurgical bacterial attachment and adhesion require a matter of hours to develop, while aggregation and dispersion can occur as early as few days after the initial attachment. ${ }^{49}$

Fig. 2D illustrates the results obtained by fitting experimental data with the analytical models selected for this study (see Data fitting). The $t^{0.5}$ dependence, indicative of a Fickian diffusion, was validated by considering the first $60 \%$ of elution, ${ }^{37,38}$ finding a satisfactory correlation between experimental data and the fitting (Fig. 2D), ultimately confirming previous results with different non-eroding nanoporous surfaces. ${ }^{14,39,40}$ In fact, in the early stages of elution, the drug is diffusing into a semi-infinite medium with zero initial concentration and thus a Fickian diffusion (i.e. $M(t) / M_{\infty}=k t^{n}$ with $\left.n=0.5\right)$ is expected. The release rate constant $k$ (i.e. slope of the fitting line in Fig. 2D) calculated at the three different pHs confirmed that the three experimental groups have a similar kinetics at the beginning of the release.

To characterize the elution kinetics until completion, we applied a model that accounts for a slower second phase resulting from the elution of molecules through interconnected channels (Fig. 2E). ${ }^{41}$ The parameter $D$, indicative of how fast the curves approach an horizontal plateau, ${ }^{14}$ showed similar values $(0.5 \pm 0.2,0.4 \pm 0.2$ and $0.4 \pm 0.1$ for $\mathrm{pH} 3,5$ and 7.4, respectively), thereby confirming that the similarities in the elution profiles at different pHs also extend to the second phase. When compared to the $D$ values previously calculated for the nanoporous titanium surface generated by oxidative nanopatterning (i.e. $1.3 \pm 0.2){ }^{14}$ these results further substantiate a slower elution. However, when compared to non-porous eluting systems such as bulk bone cement modified by hydroxyethyl methacrylate (HEMA), ${ }^{41}$ as expected the parameter $D$ of nanospongy substrates indicates a more rapid release.

In order to probe the physicochemical characteristics of the coating and ensure that a successful deposition occurred, we exploited ATR-FTIR spectroscopy to investigate the structural properties of the blend. ${ }^{36,42,43}$ Fig. 3 displays representative normalized infrared spectra of CH:PEG blend without (black line) and with genipin (orange line, $\mathrm{CH}: \mathrm{PEG}+\mathrm{GEN}$ ). The seven main bands observed were: (i) $\mathrm{O}-\mathrm{H}+\mathrm{C}-\mathrm{O}-\mathrm{H}$ stretching $\left(\nu_{\mathrm{O}-\mathrm{H}}+\nu_{\mathrm{C}-\mathrm{O}-\mathrm{H}}\right)$ in chitosan and PEG, (ii) Amide III in chitosan and/or C-O stretching $\left(\nu_{\mathrm{C}-\mathrm{O}}\right)$ in chitosan and PEG, (iii) $\mathrm{O}-\mathrm{H}$ and $\mathrm{C}-\mathrm{O}-\mathrm{H}$ 


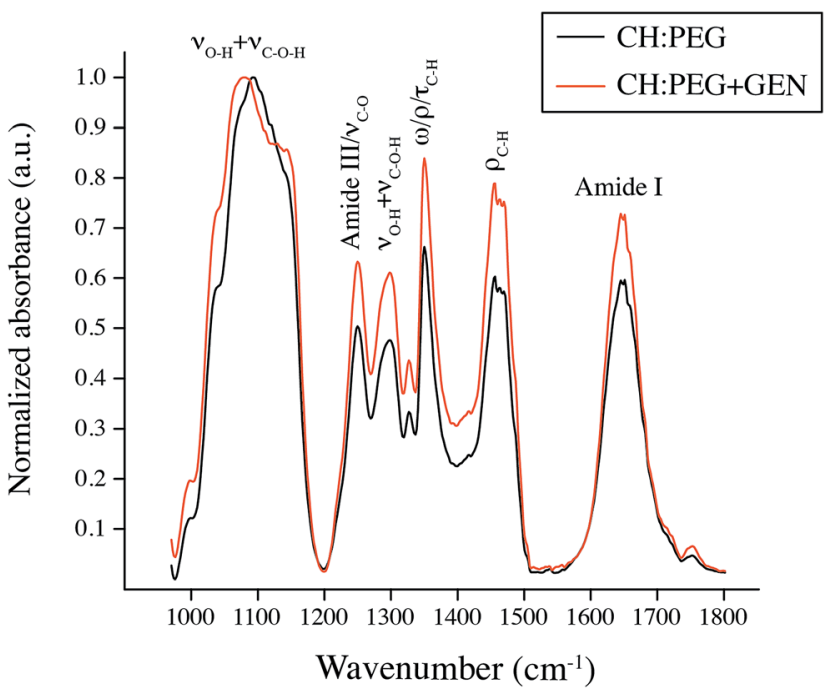

Fig. 3 Representative ATR-FTIR spectra of the uncross-linked (black solid line) and cross-linked (orange solid line) $\mathrm{CH}$ :PEG blend.

stretching $\left(\nu_{\mathrm{O}-\mathrm{H}}+\nu_{\mathrm{C}-\mathrm{O}-\mathrm{H}}\right)$ in $\mathrm{PEG}$, (iv) $\mathrm{C}-\mathrm{H}$ deformations (i.e. wagging $\omega_{\mathrm{C}-\mathrm{H}}$, bending $\rho_{\mathrm{C}-\mathrm{H}}$ and twisting $\tau_{\mathrm{C}-\mathrm{H}}$ ) in PEG, (v) C-H bending $\left(\rho_{\mathrm{C}-\mathrm{H}}\right)$ in PEG and (vi) Amide I in chitosan. During peak assignment, we were not able to resolve the Amide II band since it overlapped with the Amide I, a phenomenon previously observed in cases where the content of chitosan in the blend is below $20 \%{ }^{36}$ Table 1 reports the infrared band assignment.

While the majority of the bands are in common between chitosan and PEG, the Amide I and III only belong to the former, and was thus used to detect its presence in the blend. ${ }^{36}$ It follows that, because the infrared spectrum of the blend show the characteristic features of pure PEG (i.e. $\nu_{\mathrm{O}-\mathrm{H}}+\nu_{\mathrm{C}-\mathrm{O}-\mathrm{H}}, \omega_{\mathrm{C}-\mathrm{H}}$, $\rho_{\mathrm{C}-\mathrm{H}}$ and $\left.\tau_{\mathrm{C}-\mathrm{H}}\right)^{43}$ with the addition of the two Amide bands, we can deduce the co-existence of the two components, therefore qualitatively demonstrating the effective deposition of the CH:PEG blend onto nanospongy samples. Similarly, aside from observing the characteristic color change from transparent to blue, ${ }^{30}$ we validated the presence of genipin (chemical formula $\left.\mathrm{C}_{11} \mathrm{H}_{14} \mathrm{O}_{5}\right)^{50}$ in the cross-linked blend by detecting a relative increase of the intensity of the bands associated to the additional $\mathrm{C}-\mathrm{O}, \mathrm{C}-\mathrm{H}$ and $\mathrm{C}-\mathrm{O}$ bonds introduced into the polymeric network by the addition of the cross-linking agent (Fig. 3, orange line). Interestingly, a redshift of the band centered at about $1095 \mathrm{~cm}^{-1}\left(\nu_{\mathrm{O}-\mathrm{H}}+\nu_{\mathrm{C}-\mathrm{O}-\mathrm{H}}\right)$ was also observed upon addition of genipin. In particular, fitting of infrared data revealed that this band's maxima shifted from $1095 \pm 10 \mathrm{~cm}^{-1}$ to $1070 \pm$

Table 1 Infrared band assignment

\begin{tabular}{lll}
\hline Bands & Center $\left(\mathrm{cm}^{-1}\right)$ & References \\
\hline$\nu_{\mathrm{O}-\mathrm{H}}+\nu_{\mathrm{C}-\mathrm{O}-\mathrm{H}}$ & $\sim 1095$ & 43 \\
Amide III $/ \nu_{\mathrm{C}-\mathrm{O}}$ & 1250 & 36 and 43 \\
$\nu_{\mathrm{O}-\mathrm{H}}+\nu_{\mathrm{C}-\mathrm{O}-\mathrm{H}}$ & 1290 & 43 \\
$\omega_{\mathrm{C}-\mathrm{H}} / \rho_{\mathrm{C}-\mathrm{H}} / \tau_{\mathrm{C}-\mathrm{H}}$ & 1327,1350 & 36 and 43 \\
$\rho_{\mathrm{C}-\mathrm{H}}$ & 1460 & 43 \\
Amide I & 1646 & 36
\end{tabular}

$7 \mathrm{~cm}^{-1}$. This phenomenon may be attributed to the rupture and rearrangement of intermolecular hydrogen bonds between chitosan's hydroxyl groups and PEG's C-O-C groups ${ }^{36}$ as a consequence of the creation of crosslinking bridges of short chains of condensed genipin across the polymeric mesh. ${ }^{30}$

Infrared analysis was complemented by DPFM-AFM investigation to close in on the nanotopographical and nanomechanical features of the CH:PEG coating. Fig. 4 displays representative $5 \times 5 \mu \mathrm{m}^{2}$ DPFM-AFM micrographs of the CH:PEG blend, both uncross-linked (Fig. 4A) and cross-linked (Fig. 4B). Both conditions showed a granular morphology characteristic of chitosan- and PEG-based hydrogels. ${ }^{36,51,52}$ However, in the case of the cross-linked blend, the granular features resulted significantly finer, suggesting a more compact disposition of polymeric chains. In addition, DPFM-AFM permitted to compare the effects of genipin on the stiffness of the coating. In particular, the uncross-linked blend exhibited an average stiffness of $2.9 \pm 0.5 \mathrm{~N} \mathrm{~m}^{-1}$ (Fig. 4C), a value in good agreement with previous nanomechanical AFM measurements on PEG-based hydrogels. ${ }^{53}$ In the case of the cross-linked blend, a slightly higher value of $3.1 \pm 0.5 \mathrm{~N} \mathrm{~m}^{-1}$ was measured (Fig. 4D). Fig. 4C and D also show that the stiffness' minima and maxima increased as a consequence of the addition of genipin (i.e. from $2.5 \pm 0.2$ to $2.6 \pm 0.2 \mathrm{~N} \mathrm{~m}^{-1}$ and from $3.4 \pm 0.2$ to $3.5 \pm$ $0.1 \mathrm{~N} \mathrm{~m}^{-1}$, respectively). Normality and two-sample $t$-test ensured that such differences were statistically significant $(p<$ 0.05). Taken together, spectroscopic, morphological and nanomechanical results indicate a rearrangement of neighboring chains into a more compact polymer network provoked by the cross-linking. More importantly, such structural chances are also expected to affect the release of vancomycin by decreasing the space available in the polymeric mesh and hamper the diffusion of the antibiotic molecules. ${ }^{28}$

Fig. 5 displays the stability of the uncross-linked (Fig. 5A) and cross-linked blend (Fig. 5B) in aqueous solutions at different pHs. In both cases, the majority of the coating dissolves rapidly because of the solubility of PEG in water and that of chitosan in an acidic environment. ${ }^{36}$ The addition of genipin permitted to achieve a slightly better overall stability, ${ }^{33}$ especially at shorter intervals ( $<1$ hour). In the case of the uncross-linked blend, a pH-dependent dissolution characterized by an increasingly faster rate at $\mathrm{pH}=3$ was observed after 24 hours. Conversely, degradation of the coating at pH 5 and 7.4 appeared to plateau. Such trend was not observed in the crosslinked blend. Dissolution in fact stabilized after one hour and exhibited a similar behavior for the three conditions tested as a result of the action of genipin which offset the dependence on the $\mathrm{pH}$ by bridging polymeric chains together. From the stability data, we confirmed that the selected CH:PEG coatings provide the sought antifouling capacities to hamper the initial bacterial attachment via dissolution. Such degradation becomes $\mathrm{pH}$-responsive in the longer term ( $>24$ hours) for the uncross-linked blend. Noteworthy, despite such initial dissolution, enough polymeric material (i.e. approximately $3 \mathrm{mg}$ ) is retained onto the disks to exert $\mathrm{pH}$-sensitive effects on the elution of vancomycin (Fig. 5E and F). 

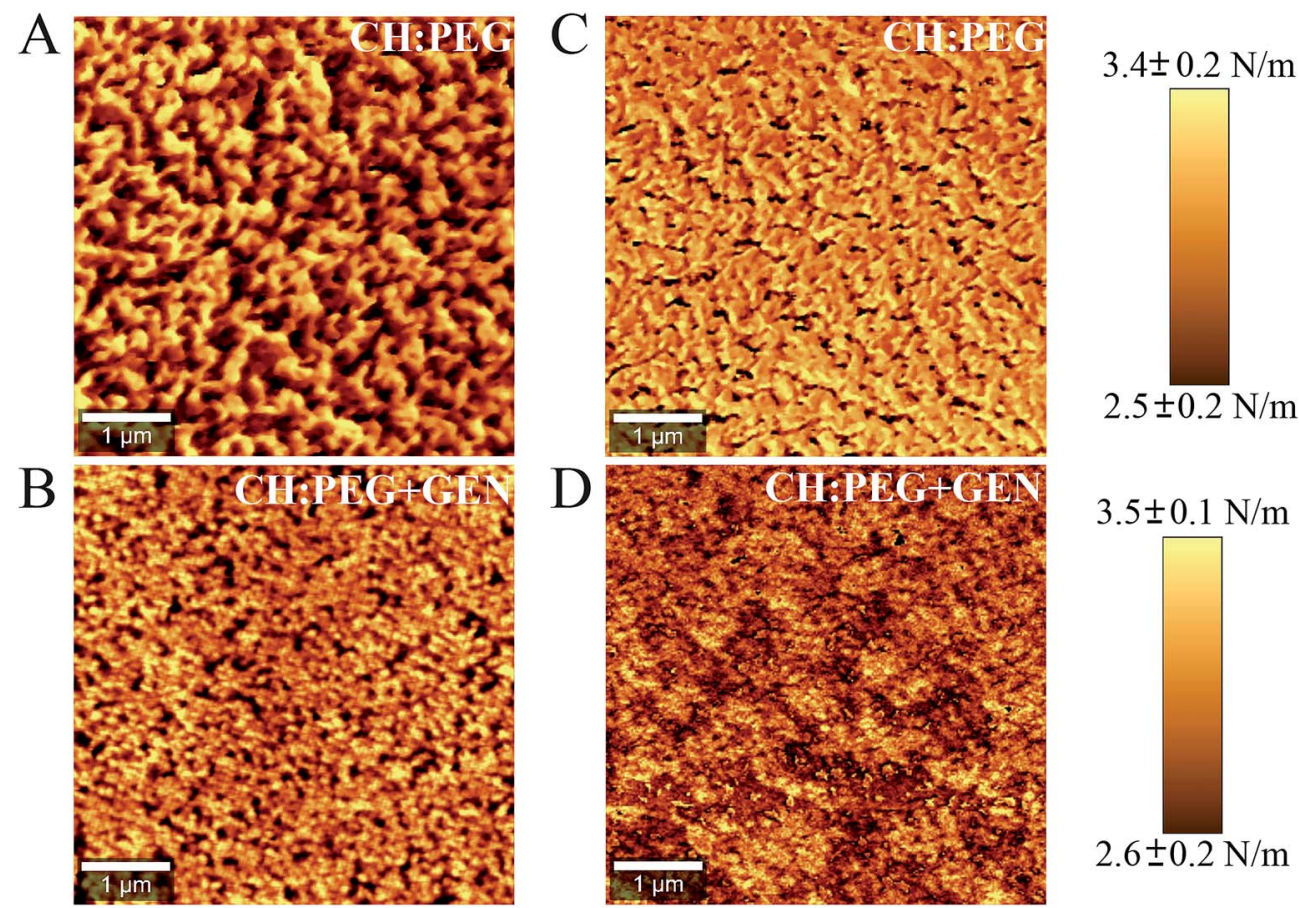

Fig. 4 Topography (left) and stiffness (right) maps obtained by AFM-DPFM on the uncross-linked (top) and cross-linked (bottom) CH:PEG blend.

The swelling characteristics of the uncross-linked and crosslinked blend at different pHs are shown in Fig. 5C and D, respectively. It can be readily observed that swelling behavior at $\mathrm{pH}=3$ diverges from that at the other two $\mathrm{pHs}$, exhibiting increasingly higher values from early intervals onwards, regardless of the presence of genipin. The greater swelling at low pHs is due to the fact that at acidic conditions the amine groups of chitosan acquire a proton, establishing repulsive forces between molecules of the same charge that ultimately cause greater intermolecular distances and hydrophilicity. ${ }^{28}$ Noteworthy, like in the case of the stability (Fig. $5 \mathrm{~A}$ and $\mathrm{B}$ ), $\mathrm{pH}$-dependent variations became more evident after 24 hours. In particular, in the case of the uncross-linked blend, from this time onwards a clear separation of the three curves occurred, indicating a marked $\mathrm{pH}^{-}$ dependent trend characterized by an increasingly pronounced swelling during acidification. In addition, while at $\mathrm{pH} 7.4$ swelling equilibrium is reached after one hour (i.e. swelling plateaus), this condition appears delayed at pHs 5 and 3, since swelling shows increasing values as a function of time. Conversely, while the behavior at $\mathrm{pH}=3$ was similar, the addition of genipin reduced the swelling at pH 5 and 7.4, offsetting, for the former, the time-dependent effects observed in the uncross-linked blend. This is consistent with the fact that a crosslinking agent hampers the relative motion of the polymeric chains, resulting in a less pronounced swelling at equilibrium. At $\mathrm{pH}=3$, our data indicate that the repulsive forces overcame the effects of genipin, and thus suggest that a higher concentration of the cross-linking agent is needed for lower pHs.

The release profiles of CH:PEG coated nanospongy surfaces are shown in Fig. 5, for the uncross-linked and cross-linked coating. In the case of the uncross-linked film (Fig. 5C), we can observe the following effects: (i) while uncoated surfaces terminate the elution after a week (Fig. 2), CH:PEG coated surfaces elute between 50 and $70 \%$ of the stored vancomycin at this time, therefore indicating a slower overall release resulting from the diffusion of the solute through the polymeric coating. (ii) By employing the uncross-linked coating, we achieved a pHdependent elution, slower at $\mathrm{pH}=7.4$ and faster at $\mathrm{pH}=3$. The higher swelling at lower $\mathrm{pH}$ is in fact expected to create a largesized mesh, resulting in a higher permeability of the film. The behavior at $\mathrm{pH}=5$ showed a trend very close to that recorded at $\mathrm{pH}$ 7.4, indicating that the minimal variations in stability and swelling between these two conditions displayed in Fig. 5A and $\mathrm{C}$ are not sufficient to induce a significantly different release profile, even after 24 hours.

In the case of the cross-linked film (Fig. 5D), the following considerations can be made: (i) the addition of genipin further delays the elution: after a week only $40-50 \%$ of vancomycin was released. This result derives from the fact that the permeability of the cross-linked blend to the diffusion of vancomycin decreased ${ }^{28}$ ultimately yielding a slower release of the antibiotics. (ii) The pH-dependent release is maintained. In fact, the elution kinetics increased in response to the acidification of the environment. Similarly to the case of the uncross-linked film, the behavior at $\mathrm{pH} 3$ and 7.4 was distinct and separate. The trend at $\mathrm{pH}=5$ did not significantly differentiate from that at $\mathrm{pH} 7.4$, as anticipated by the stability and swelling results, although a moderately faster elution can be observe for the first hour.

It should be noted that the models used to precisely quantify the elution from nanospongy surfaces could not be applied to CH:PEG coated samples, since they do not account for the solute diffusion through hydrated polymer membranes. In fact, 

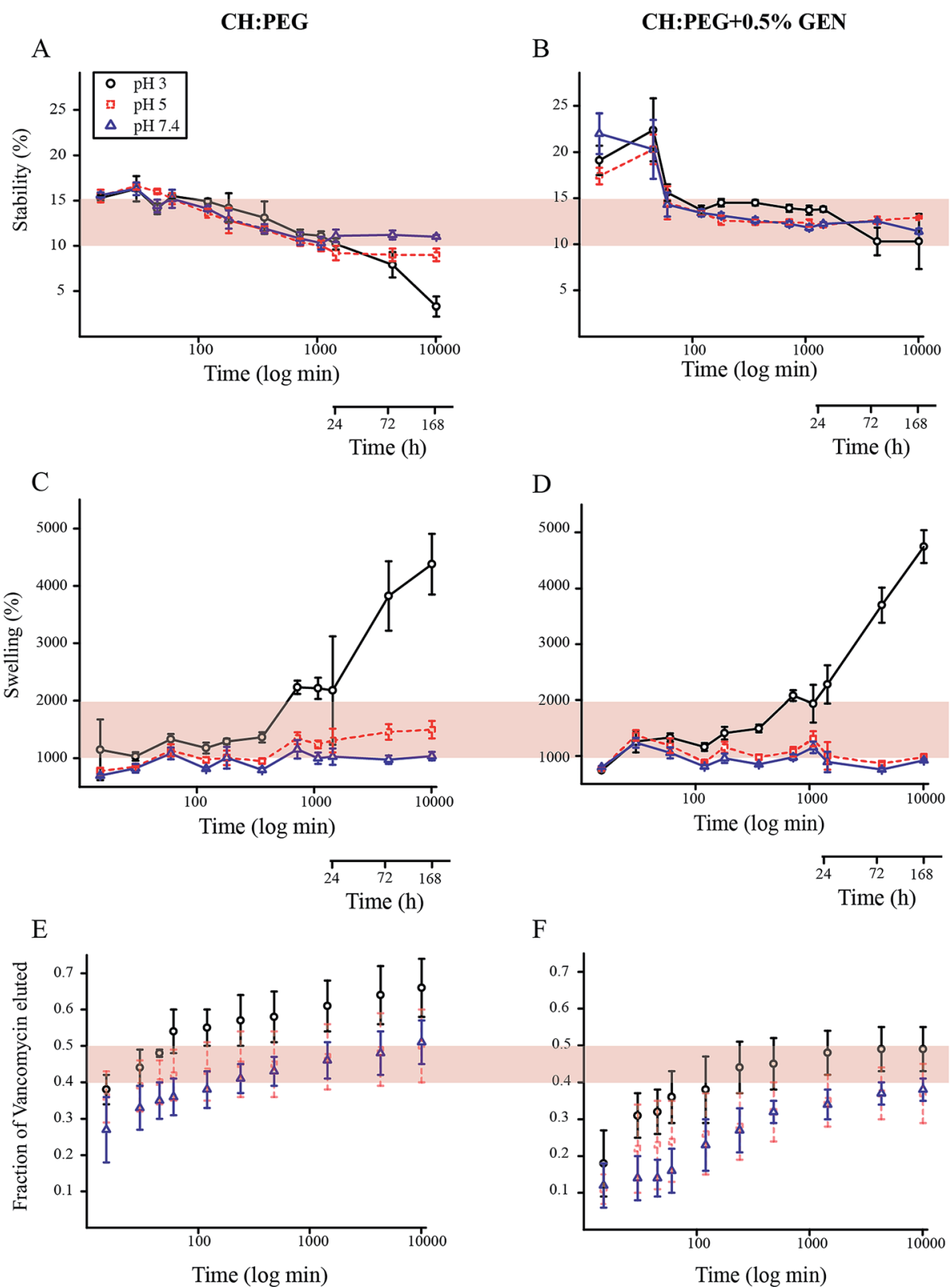

$\mathrm{F}$
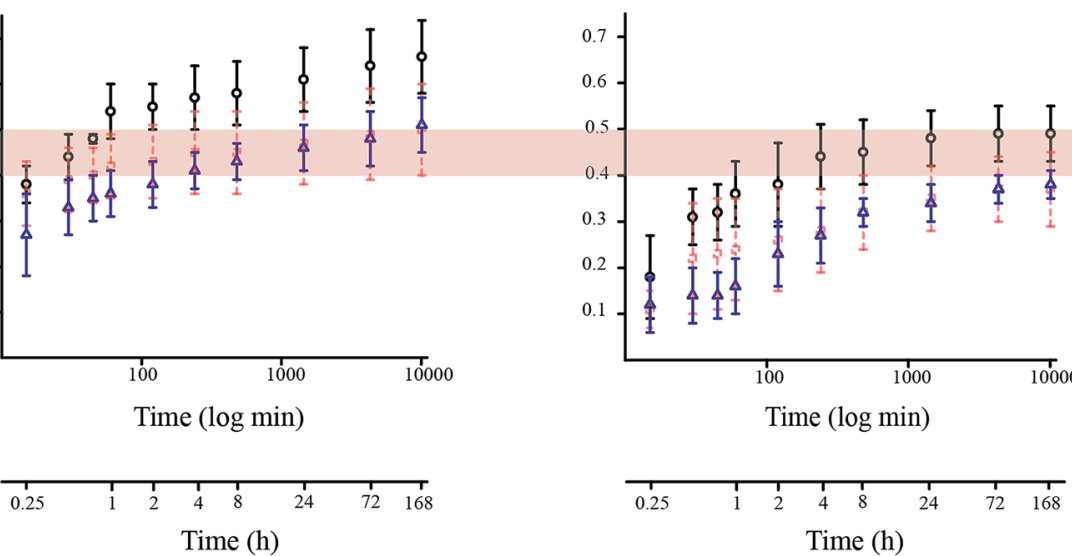

Fig. 5 (A and B) Stability in aqueous solutions at pH 3, 5 and 7.4 of the uncross-linked (left) and cross-linked (right) blend ( $n=5)$. The red shadowing indicates the 10-15\% range of stability and was used as a visual reference for comparison. (C and D) Swelling behaviour in aqueous solutions at $\mathrm{pH} 3,5$ and 7.4 of the uncross-linked (left) and cross-linked (right) blend $(n=5)$. The red shadowing indicates the $1000-2000 \%$ range of swelling and was used as a visual reference for comparison. (E and F) Point-by-point curve of the fraction eluted of vancomycin from nanospongy surfaces coated with the uncross-linked (left) and cross-linked (right) blend at pH 3, 5 and $7.4(n=5)$. Data points are visualized as mean \pm standard deviation. To enhance clarity, the elution curve at $\mathrm{pH}=5$ (red dotted line) is shown with a transparency of $50 \%$. The red shadowing indicates the $40-50 \%$ range of elution and was used as a visual reference for comparison.

in the case of CH:PEG coated surfaces, the free volume theory should be applied. ${ }^{28,54,55}$ Conversely, in the case of cross-linked $\mathrm{CH}$ :PEG blends, the models that account for the influence of crosslinking on diffusive properties should be used. ${ }^{28,56}$ However, we were not able to employ either of these models since some of the required parameters were unknown and could not be determined in this study. We therefore could not further the analysis of the release kinetics beyond the quantitative results presented in Fig. $5 \mathrm{E}$ and $\mathrm{F}$, which nonetheless demonstrate a slower and $\mathrm{pH}$-dependent release when the polymeric film is used.

In conclusion, we can assert that the main mechanism that controls the release of vancomycin is the diffusion through the film, a process which is modulated by a combination of the 
pH-dependent swelling and the cross-linking effects of genipin, the latter responsible for decreasing the mesh size of the film and, in turn, hampering to diffusion rate of the solute molecules. While these effects are strongly marked between $\mathrm{pH} 3$ and 7.4 and result in distinct and well-apart elution profiles, they are not significant to separate the behavior at pH 5 .

\section{Conclusions}

While nanoporous and nanotubular titanium have already demonstrated the ability to store drugs and modulate their elution over time, the potential of a nanospongy surface created by anodization was yet to be validated. To this end, we investigated the potential of this to act as a non-eroding drug-eluting platform. Our data show that the resulting 3-dimensional nanoporosity is capable of storing vancomycin and providing its extended elution for over one week, offering a valid alternative to existing non-eroding nanoporous surfaces for drug-delivery applications. To endow such surface with the ability of responding to a local acidification of the surrounding environment, as a proof of concept we employed a pH-sensitive $\mathrm{CH}$ :PEG blend as a "molecular filter" to regulate the release of the antibiotic. Our results show that this approach permits to add one extra level of synergistic control of the overall behaviour of the system by providing (i) a rapid initial dissolution to engender antifouling abilities and (ii) a slower and $\mathrm{pH}^{-}$ dependent release to address the localized acidification associated to bacterial infection. In this context, we considered a relatively wide range of pHs (7.4-3) to incorporate additional events characterized by a localized decrease (e.g. bone remodelling, tissue inflammation). Because of the applicability of this approach to potentially any non-eroding templates, our results open the door for future studies with different surfaces aimed at increasing the loading ability and further extend the elution. In addition, because of the ability demonstrated by chitosan-PEG coating to modulate the elution, future work can focus on tuning its physicochemical characteristics in order to provide a responsive reaction to smaller $\mathrm{pH}$ variations.

\section{Acknowledgements}

F.V. acknowledges financial support from the Natural Sciences and Engineering Research Council of Canada (NSERC) through the Discovery grant, from the Canada Foundation for Innovation (CFI) and the Ontario Ministry of Research and Innovation (MRI) through the Leaders of Opportunity (LOF) fund. S.A. and A.A. acknowledge financial support from the Natural Sciences and Engineering Research Council of Canada (NSERC) through the Undergraduate Student Research Awards (USRA) grant. We thank Davide A. D. Cassani for the AFM image in Fig. 1 and Dr Glenn A. Facey for H-NMR measurements.

\section{References}

1 G. G. Walmsley, A. McArdle, R. Tevlin, A. Momeni, D. Atashroo, M. S. Hu, A. H. Feroze, V. W. Wong, P. H. Lorenz,
M. T. Longaker and D. C. Wan, Nanomedicine, 2015, 11, 1253-1263.

2 V. Goriainov, R. Cook, J. M. Latham, D. G. Dunlop and R. O. C. Oreffo, Acta Biomater., 2014, 10, 4043-4057.

3 J. H. Lee and T. Ogawa, Implant Dent., 2012, 21, 415-421.

4 E. Korol, K. Johnston, N. Waser, F. Sifakis, H. S. Jafri, M. Lo and M. H. Kyaw, PLoS One, 2013, 8, e83743.

5 B. Ercan, E. Taylor, E. Alpaslan and T. J. Webster, Nanotechnology, 2011, 22, 295102.

6 G. Thakral, R. Thakral, N. Sharma, J. Seth and P. Vashisht, J. Clin. Diagn. Res., 2014, 8, ZE07-ZE10.

7 F. Variola, A. Lauria, A. Nanci and F. Rosei, Adv. Eng. Mater., 2009, 11, B227-B234.

8 S. Minagar, C. C. Berndt, J. Wang, E. Ivanova and C. Wen, Acta Biomater., 2012, 8, 2875-2888.

9 F. Vetrone, F. Variola, P. T. d. Oliveira, S. F. Zalzal, J.-H. Yi, J. Sam, K. F. Bombonato-Prado, A. Sarkissian, D. F. Perepichka, J. D. Wuest, F. Rosei and A. Nanci, Nano Lett., 2009, 9, 659-665.

10 A. F. Cipriano, C. Miller and H. Liu, J. Biomed. Nanotechnol., 2014, 10, 2977-3003.

11 L. R. Rodrigues, Adv. Exp. Med. Biol., 2011, 715, 351-367.

12 F. Variola, S. F. Zalzal, A. Leduc, J. Barbeau and A. Nanci, Int. J. Nanomed., 2014, 4, 2319-2325.

13 B. Ercan, K. M. Kummer, K. M. Tarquinio and T. J. Webster, Acta Biomater., 2011, 7, 3003-3012.

14 A. Ketabchi, K. Komm, M. Miles-Rossouw, D. A. D. Cassani and F. Variola, PLoS One, 2014, 9, e92080.

15 D. Losic, M. S. Aw, A. Santos, K. Gulati and M. Bariana, Expert Opin. Drug Delivery, 2015, 12, 103-127.

16 A. M. Peterson, H. Möhwald and D. G. Shchukin, Biomacromolecules, 2012, 13, 3120-3126.

17 N. Bhattarai, J. Gunn and M. Zhang, Adv. Drug Delivery Rev., 2010, 62, 83-99.

18 S. Eliyahu-Gross and R. Bitton, Rev. Chem. Eng., 2013, 29, 159-168.

19 N. Bhattarai, F. A. Matsen and M. Zhang, Macromol. Biosci., 2005, 5, 107-111.

20 T. Kowada, J. Kikuta, A. Kubo, M. Ishii, H. Maeda, S. Mizukami and K. Kikuchi, J. Am. Chem. Soc., 2011, 133, 17772-17776.

21 B. Clarke, Clin. J. Am. Soc. Nephrol., 2008, 3, S131.

22 T. Ueno, H. Tsuchiya, M. Mizogami and K. Takakura, J. Inflammation Res., 2008, 1, 41.

23 I. Zhuk, F. Jariwala, A. B. Attygalle, Y. Wu, M. R. Libera and S. A. Sukhishvili, ACS Nano, 2014, 8, 7733-7745.

24 B. Gonzalez, M. Colilla and M. A. Vallet-Regí, Chem. Mater., 2008, 20, 4826.

25 S. Pavlukhina, Y. Lu, A. Patimetha, M. Libera and S. Sukhishvili, Biomacromolecules, 2010, 11, 3448-3456.

26 A. Ketabchi, A. Weck and F. Variola, J. Biomed. Mater. Res., Part B, 2015, 103, 563-571.

27 F. Ordikhani, E. Tamjid and A. Simchi, Mater. Sci. Eng., C, 2014, 41, 240-248.

28 J. Jin and M. Song, J. Appl. Polym. Sci., 2006, 102, 436-444.

29 K. Gulati, S. Ramakrishnan, M. S. Aw, G. J. Atkins, D. M. Findlay and D. Losic, Acta Biomater., 2012, 8, 449-456. 
30 R. A. A. Muzzarelli, Carbohydr. Polym., 2009, 77, 1-9.

31 X. Song, H. Wu, S. Li, Y. Wang, X. Ma and M. Tan, Biomacromolecules, 2015, 16, 2080-2090.

32 S. Zivanovic, J. Li, P. M. Davidson and K. Kit, Biomacromolecules, 2007, 8, 1505-1510.

33 J. Jin, M. Song and D. J. Hourston, Biomacromolecules, 2004, 5, 162-168.

34 G. L. Ackland, A. G. D. Arroyo, S. T. Yao, R. C. Stephens, A. Dyson, N. J. Klein, M. Singer and A. V. Gourine, Crit. Care Med., 2010, 38, 629-636.

35 C. A. Rezende, L.-T. Lee and F. Galembeck, Langmuir, 2009, 25, 9938-9946.

36 D. A. D. Cassani, L. Altomare, L. D. Nardo and F. Variola, J. Mater. Chem. B, 2015, 3, 2641-2650.

37 P. L. Ritger and N. A. Peppas, J. Controlled Release, 1987, 5, 23-36.

38 H. R. Aucoin, A. N. Wilson, A. M. Wilson, K. Ishihara and A. Guiseppi-Elie, Polymer, 2013, 5, 1241-1257.

39 E. Gultepe, D. Nagesha, B. D. F. Casse, R. Banyal, T. Fitchorov, A. Karma, M. Amiji and S. Sridhar, Small, 2010, 6, 213-216.

40 L. Peng, A. D. Mendelsohn, T. J. LaTempa, S. Yoriya, C. A. Grimes and T. A. Desai, Nano Lett., 2009, 9, 1932-1936.

41 P. Frutos, E. Diez-Pena, G. Frutos and J. M. Barrales-Riendaa, Biomaterials, 2002, 23, 3787-3797.

42 M. F. Butler, Y.-F. Ng and P. D. A. Pudney, J. Polym. Sci., Part A: Polym. Chem., 2003, 41, 3941-3953.

43 K. Shameli, M. B. Ahmad, S. D. Jazayeri, S. Sedaghat, P. Shabanzadeh, H. Jahangirian, M. Mahdavi and Y. Abdollahi, Int. J. Mol. Sci., 2012, 13, 6639-6650.
44 C. A. Schneider, W. S. Rasband and K. W. Eliceiri, Nat. Methods, 2012, 9, 671-675.

45 R. Sánchez-Tovar, K. Lee, J. Garciía-Antón and P. Schmuki, Electrochem. Commun., 2013, 26, 1-4.

46 K. S. Brammer, S. Oh, C. J. Cobb, L. M. Bjursten, H. v. d. Heyde and S. Jin, Acta Biomater., 2009, 5, 3215-3223.

47 J.-H. Yi, C. Bernard, F. Variola, S. F. Zalzal, J. D. Wuest, F. Rosei and A. Nanci, Surf. Sci., 2006, 600, 4613-4621.

48 T. E. Swanson, X. Cheng and C. Friedrich, J. Biomed. Mater. Res., Part A, 2011, 97, 167-176.

49 J. S. Temenoff and A. G. Mikos, Biomaterials: the intersection of biology and materials science, Pearson Prentice Hall, 2008.

50 S. D. Tommaso, P. David, K. Picolet, M. Gabant, H. David, J.-L. Morançais, J. Gomar, F. Leroy and C. Adamo, RSC Adv. , 2013, 3, 13764-13771.

51 Q. Huang, I. Yoon, J. Villanueva, K. Kimb and D. J. Sirbuly, Soft Matter, 2014, 10, 8001-8010.

52 S. Sharma, R. W. Johnson and T. A. Desai, Biosens. Bioelectron., 2004, 20, 227-239.

53 Z. Drira and V. K. Yadavalli, J. Mech. Behav. Biomed. Mater., 2013, 18, 20-28.

54 H. Yasuda, C. E. Lamaze and L. D. Ikenberry, Makromol. Chem., 1968, 118, 19-35.

55 H. Yasuda, A. Peterlin, C. K. Colton, K. A. Smith and E. W. Merrill, Makromol. Chem., 1969, 126, 177-186.

56 C. T. Reinhart and N. A. Peppas, J. Membr. Sci., 1984, 18, 227239. 